INPLASY

PROTOCOL

To cite: Kuang et al. Efficacy and safety of acupuncture combined with analgesics on lung cancer pain: A protocol for systematic review and meta-analysis. Inplasy protocol 202150051. doi:

10.37766/inplasy2021.5.0051

Received: 14 May 2021

Published: 14 May 2021

Corresponding author:

Renqing Kuang

1556338577@qq.com

Author Affiliation:

Jiangxi University of Chinese Medicine

Support: Grant No.2019A140.

Review Stage at time of this submission: Preliminary

searches.

Conflicts of interest:

None declared.

\section{Efficacy and safety of acupuncture combined with analgesics on lung cancer pain: A protocol for systematic review and meta-analysis}

Kuang, RQ1; Xiong, GJ2; Lv, W3; Zhao, Y4; Yu, M5; Jiang, JW6.

Review question / Objective: Efficacy and safety of acupuncture combined with analgesics on lung cancer pain. Condition being studied: Lung cancer (LC) is the malignant tumor with the highest incidence in the world, and treatment methods include surgery, radiotherapy, chemotherapy and immunotherapy. Cancer pain is a common symptom in patients with LC, and the clinical treatment is to relieve it with analgesics. Acupuncture can relieve cancer pain. This study aims to systematically study the efficacy and safety of acupuncture combined with analgesics on cancer pain in patients with LC.

Information sources: The English databases include PubMed, Embase, Web of Science, Cochrane Library, and Chinese databases include China National Knowledge Infrastructure (CNKI), Wanfang Data, VIP Database (VIP), China Biomedical Literature (CBM). From the establishment of the database to April, 2021. The key words include "acupuncture", "body acupuncture", "electro-acupuncture", "warm acupuncture", "auricular acupuncture", "fire needling", "analgesics", "lung cancer", "pain". In addition, we will also retrieve ongoing or unpublished trials from the International Clinical Trial Registration Platform and Chinese Clinical Trial Registry Platform.

INPLASY registration number: This protocol was registered with the International Platform of Registered Systematic Review and Meta-Analysis Protocols (INPLASY) on 14 May 2021 and was last updated on 14 May 2021 (registration number INPLASY202150051).

\section{INTRODUCTION}

Review question / Objective: Efficacy and safety of acupuncture combined with analgesics on lung cancer pain.
Condition being studied: Lung cancer (LC) is the malignant tumor with the highest incidence in the world, and treatment methods include surgery, radiotherapy, chemotherapy and immunotherapy. Cancer 
pain is a common symptom in patients with LC, and the clinical treatment is to relieve it with analgesics. Acupuncture can relieve cancer pain. This study aims to systematically study the efficacy and safety of acupuncture combined with analgesics on cancer pain in patients with LC.

\section{METHODS}

Participant or population: All the patients included in the study were diagnosed LC pain, regardless of age, sex, race and course of disease.

Intervention: The treatment plan is acupuncture combined with analgesics. Acupuncture treatment includes all types, such as electric acupuncture, fire acupuncture, warm acupuncture, body acupuncture and so on.

\section{Comparator: Conventional analgesic treatment.}

\section{Study designs to be included: RCT.}

Eligibility criteria: 1. All RCTs of acupuncture combined with analgesics in the treatment of LC pain, whether blinded or unblinded. 2. All the patients included in the study were diagnosed LC pain, regardless of age, sex, race and course of disease. 3. The treatment plan is acupuncture combined with analgesics.

Information sources: The English databases include PubMed, Embase, Web of Science, Cochrane Library, and Chinese databases include China National Knowledge Infrastructure (CNKI), Wanfang Data, VIP Database (VIP), China Biomedical Literature (CBM). From the establishment of the database to April, 2021. The key words include "acupuncture", "body acupuncture", "electro-acupuncture", "warm acupuncture", "auricular acupuncture", "fire needling", "analgesics", "lung cancer", "pain". In addition, we will also retrieve ongoing or unpublished trials from the International Clinical Trial Registration Platform and Chinese Clinical Trial Registry Platform.
Main outcome(s): The main outcome measure was the total effective rate of analgesia (excluding the evaluation of inefficiency and non-remission rate).

Quality assessment / Risk of bias analysis: Two reviewers will use Cochrane collaborative tools to evaluate the quality of the literature. It includes the following 7 domains: random sequence generation, allocation concealment, blinding of participants and personnel, blinding of outcome assessment, incomplete outcome data, selective reporting and other sources of bias. Each domain includes a judgment of low, high and unclear risk of bias according to information provided by authors. Disagreements between reviewers will be resolved through discussion with a third reviewer.

Strategy of data synthesis: For continuous data, will be used mean difference (MD) as the effect indicator with $95 \%$ confidence interval, and dichotomous data will be calculated as risk ratio (RR) or odds ratio (OR)as the effect index with $95 \%$ confidence interval. If the studies with no statistical homogeneity, the fixed-effect model can be used for analysis; if the studies with significant statistical heterogeneity, random effects model analysis will be used. The Review Manager (RevMan) V.5.3 software will be used for statistical analysis.

Subgroup analysis: Subgroup analysis will be performed if there have heterogeneity between the study results, following items will be considered: type of acupuncture, cancer stage, gender, age.

Sensitivity analysis: We will eliminate the "high-risk" low-quality articles for sensitivity analysis to judge the robustness of the results.

Country(ies) involved: China.

Keywords: acupuncture, lung cancer, pain, protocol, systematic review. 
Contributions of each author:

Author 1 - Renqing Kuang.

Email: 1556338577@qq.com

Author 2 - Guojiang Xiong.

Email: 1160888698@qq.com

Author 3 - Wei Lv.

Email: 125438851@qq.com

Author 4 - Yun Zhao.

Email: 919302219@qq.com

Author 5 - Min Yu.

Email: 1063807362@qq.com

Author 6 - Jiawang Jiang.

Email: 1137298371@qq.com 